\title{
Metamodulation of a Spinal Locomotor Network by Nitric Oxide
}

\author{
David L. McLean and Keith T. Sillar \\ Division of Biomedical Sciences, School of Biology, University of St Andrews, St Andrews, Fife KY16 9TS, United Kingdom
}

Flexibility in the output of spinal networks can be accomplished by the actions of neuromodulators; however, little is known about how the process of neuromodulation itself may be modulated. Here we investigate the potential "meta"-modulatory hierarchy between nitric oxide (NO) and noradrenaline (NA) in Xenopus laevis tadpoles. NO and NA have similar effects on fictive swimming; both potentiate glycinergic inhibition to slow swimming frequency and GABAergic inhibition to reduce episode durations. In addition, both modulators have direct effects on the membrane properties of motor neurons. Here we report that antagonism of noradrenergic pathways with phentolamine dramatically influences the effect of the NO donor $S$-nitroso- $N$-acetylpenicillamine (SNAP) on swimming frequency, but not its effect on episode durations. In contrast, scavenging extracellular NO with 2-(4-carboxyphenyl)-4,4,5,5-tetramethylimidazoline1-oxyl-3-oxide (PTIO) does not influence any of the effects of NA on fictive swimming. These data place NO above NA in the metamodulatory hierarchy, strongly suggesting that NO works via a noradrenergic pathway to control glycine release but directly promotes GABA release. We confirmed this possibility using intracellular recordings from motor neurons. In support of a natural role for NO in the Xenopus locomotor network, PTIO not only antagonized all of the effects of SNAP on swimming but also, when applied on its own, modulated both swimming frequency and episode durations in addition to the underlying glycinergic and GABAergic pathways. Collectively, our results illustrate that NO and NA have parallel effects on motor neuron membrane properties and GABAergic inhibition, but that NO serially metamodulates glycinergic inhibition via NA.

Key words: nitric oxide; noradrenaline; inhibition; brainstem; spinal cord; locomotion; Xenopus laevis

\section{Introduction}

To survive, all animals must interact with their surroundings by responding appropriately to sensory cues. For vertebrate locomotion, this demands flexibility in the output of networks located in the spinal cord. One way locomotor networks are made more adaptable is by neuromodulators, which change the strength of synaptic connections and/or modify the integrative membrane properties of component neurons (Kiehn et al., 1997; Sillar et al., 1997). As neuromodulatory mechanisms continue to be elucidated (Harris-Warrick and Marder, 1991; Simmers et al., 1995; Marder and Calabrese, 1996; Katz, 1999; Nusbaum et al., 2001), more recent attention has focused on how neuromodulators themselves may be modulated (Katz and Edwards, 1999; Mesce, 2002). Such "meta"-modulatory mechanisms could reflect higher-order, integrative behavioral functions, yet very little is known about metamodulation in vertebrates (Svensson et al., 2001).

Here, we examine metamodulation in an established vertebrate model, the tadpole of the frog Xenopus laevis. From late

\footnotetext{
Received 0ct. 28, 2003; revised Sept. 9, 2004; accepted Sept. 10, 2004.

We thank The Wellcome Trust for generous support and Prof. Anthony Butler and colleagues (University of St Andrews, St Andrews, UK) for providing our SNAP. We are also extremely grateful to Dr. Simon Merrywest for his invaluable assistance during the preparation of this manuscript.

Correspondence should be addressed to Keith T. Sillar at the above address. E-mail: kts1@st-andrews.ac.uk.

D. L. McLean's current address: Department of Neurobiology and Behavior, Cornell University, Seeley Mudd Hall, Ithaca, NY 14853

D0I:10.1523/JNEUROSCI.1817-04.2004

Copyright $\odot 2004$ Society for Neuroscience $\quad$ 0270-6474/04/249561-11\$15.00/0
}

embryonic to early larval stages (stage 37/38-42) (Nieuwkoop and Faber, 1956), Xenopus tadpoles swim primarily in response to sensory stimulation. During swimming, waves of axial muscle contractions alternate across the body and pass from head to tail, propelling the animal forward. The neural circuitry driving this behavior is relatively simple (Roberts, 1990). The frequency of swimming is primarily determined by the balance between synaptic excitation (Dale and Roberts, 1985; Sillar and Roberts, 1993; Perrins and Roberts, 1995) and inhibition (Soffe, 1987; Dale, 1995; Reith and Sillar, 1999). Once initiated, swimming will continue until either the animal's head contacts an obstacle and cement gland afferents trigger inhibitory GABA release from reticulospinal neurons onto spinal neurons (Boothby and Roberts, 1992a,b; Perrins et al., 2002; Li et al., 2003), or locomotion gradually runs down as a result of adenosine accumulation (Dale and Gilday, 1996; Dale, 2002).

How fast and how far the tadpole swims are two facets of locomotion that are major targets for neuromodulation (Sillar et al., 2002). For example, the amines serotonin [5-hydroxytryptamine (5-HT)] and noradrenaline (NA) exert opposing modulatory influences over the amplitude of midcycle inhibition by decreasing or increasing, respectively, glycinergic inhibition from commissural interneurons onto motor neurons (McDearmid et al., 1997). Another neurotransmitter that has recently been shown to affect inhibitory synaptic transmission in the spinal cord of Xenopus is nitric oxide (NO). Like NA, NO increases midcycle glycinergic inhibition while simultaneously increasing 

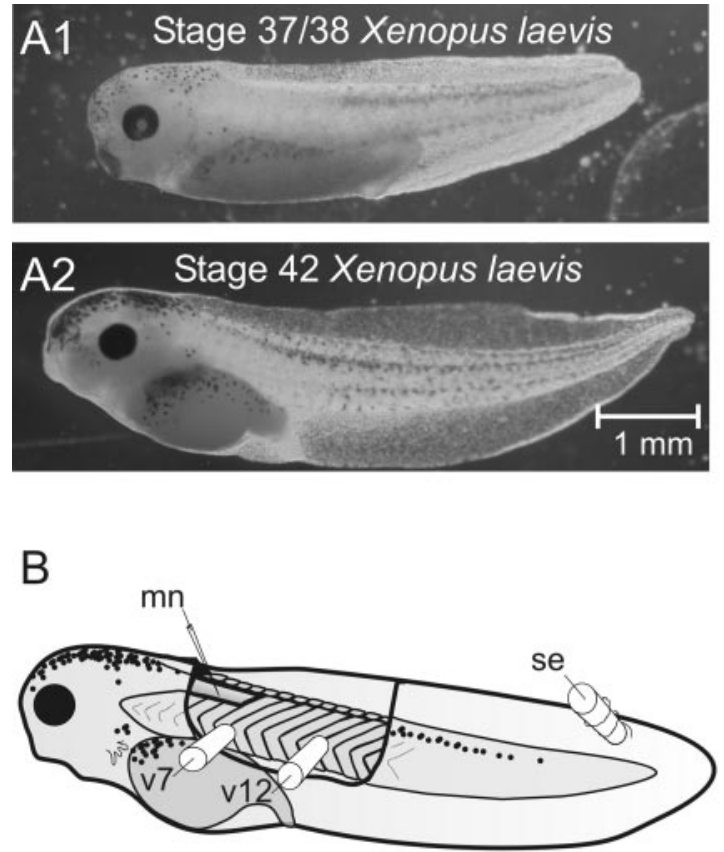
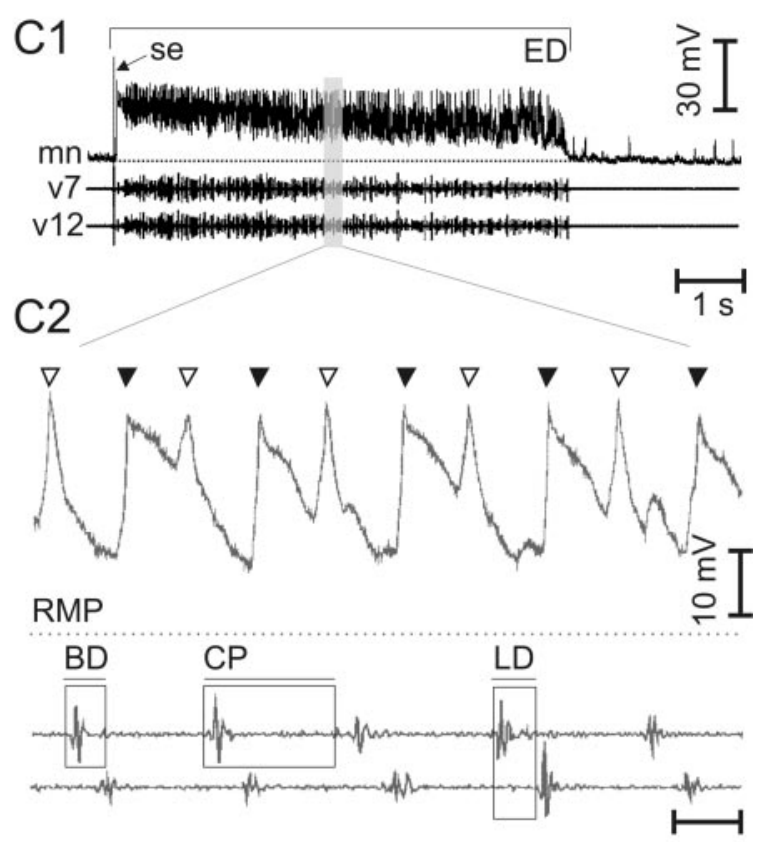

$25 \mathrm{~ms}$

Figure 1. The experimental setup. A, Photographs of embryos (A1; stage 37/38) and larvae (A2; stage 42) of the South African clawed frog Xenopus laevis, staged according to Nieuwkoop and Faber (1956). B, The intermyotomal clefts (as numbered from the otic capsule) and spinal cord were exposed to facilitate ventral root recordings with ipsilaterally placed electrodes (postotic clefts v7, v12) and recordings from motor neurons with an intracellular microelectrode (mn). Fictive swimming from immobilized tadpoles was elicited by a stimulating electrode (se). See Materials and Methods for more details. C, The fictive swimming rhythm at slow (C1) and fast (C2) time scales illustrates that on-cycle excitation $(\nabla)$ just precedes ipsilateral ventral root activity and midcycle inhibition $(\boldsymbol{\nabla})$, both of which are superimposed on a gradually declining tonic depolarization. KCl-filled electrodes render chloride-dependent inhibition depolarizing (see Materials and Methods). RMP, Resting membrane potential; BD, burst duration; CP, cycle period; LD, longitudinal delay; ED, episode duration. Note the stimulation artifact (se) arrowed in C1.

the occurrence of GABA IPSPs, which can terminate swimming episodes (McLean and Sillar, 2002). In addition, NO has direct effects on the membrane properties of spinal neurons (McLean and Sillar, 2002). However, at these stages, NO is not generated by neurons in the spinal cord but by neurons in close proximity to descending sources of 5-HT, NA, and GABA in the brainstem (McLean and Sillar, 2000, 2001; Lopéz and González, 2002). It is therefore tempting to suggest that some of the effects of NO are attributable to metamodulatory influences on descending aminergic systems. We provide here direct physiological evidence linking NO with the amines during vertebrate locomotion by examining the hierarchical relationship between NO and NA.

\section{Materials and Methods}

All experiments were performed on prefeeding Xenopus laevis tadpoles obtained after weekly breeding from a laboratory colony and raised in dechlorinated tap water between 17 and $23^{\circ} \mathrm{C}$ to stagger their development. Embryos and larvae (Fig. $1 A 1, A 2)$ were staged according to Nieuwkoop and Faber (1956) and anesthetized in tricaine methanesulphonate (MS-222) (0.1-1\%; Sigma-RBI, Poole, UK) before experimentation. All procedures conformed to United Kingdom Home Office regulations regarding animal experimentation as described in the Animals (Scientific Procedures) Act, 1986.

Electrophysiological recordings. After anesthesia, tadpoles were removed from the MS-222, scored along the dorsal fin with a custometched tungsten dissecting pin, and immobilized by immersion in the neuromuscular blocking agent $\alpha$-bungarotoxin (12.5 $\mu$; Sigma-RBI). Animals were removed from the toxin after determining immobilization was effective $(\sim 30-60 \mathrm{~min})$ and pinned down through the notocord to a rotateable Sylgard-coated (Dow-Corning, Midland, MI) platform within an experimental chamber through which $100 \mathrm{ml}$ of saline was constantly recirculating $(\sim 5 \mathrm{ml} / \mathrm{min})$ at room temperature [saline composition (in mM): $115 \mathrm{NaCl}, 2.5 \mathrm{KCl}, 2.5 \mathrm{NaHCO}_{3}, 10 \mathrm{HEPES}, 1 \mathrm{MgCl}_{2}$, and $2 \mathrm{CaCl}_{2}$ (for extracellular experiments) or $4 \mathrm{CaCl}_{2}$ (for intracellular experiments), $\mathrm{pH} 7.4$ with $\mathrm{NaOH}$ ]. The flank skin from the level of the otic capsule to the anus was then carefully removed with fine forceps and dissecting pins (Fig. $1 \mathrm{~B}$ ). To provide access to the spinal cord for intracellular recordings, the dorsal portion of the first few myotomes (as numbered from the otic capsule) was removed. Extracellular recordings of ventral root impulses appropriate to drive swimming movements ("fictive" swimming) were made using glass suction electrodes fashioned from borosilicate nonfilamented glass placed over the intermyotomal clefts. Intracellular recordings from presumed motor neurons in the ventral quarter of the spinal cord (Roberts and Clarke, 1982) were made using filamented glass sharp microelectrodes filled with $3 \mathrm{M} \mathrm{KCl}$ (direct current resistances, $\sim 90-120 \mathrm{M} \Omega$ ). Penetrations of cells were achieved by capacity overcompensation. In the period after initial penetration and during the establishment of the resting potential, chloride-dependent IPSPs reversed in sign to become strongly depolarizing as a result of chloride loading of the cell (Fig. 1C1,C2). In these conditions, spontaneously occurring IPSPs mediated by glycine and $\mathrm{GABA}_{\mathrm{A}}$ receptors can be readily distinguished on the basis of their durations ( $\leq 90 \mathrm{msec}$, glycine; $\geq 90 \mathrm{msec}, \mathrm{GABA}_{\mathrm{A}}$ ) and their pharmacological sensitivities (Reith and Sillar, 1997). Previous work indicates that excitatory PSPs do not contribute significantly to the spontaneous PSPs observed at these stages of development (Reith and Sillar, 1997; McLean and Sillar, 2002). Only once the membrane potential had stabilized at $-55 \mathrm{mV}$ or below and the IPSPs did not appear to vary significantly between episodes were experiments begun. Changes in amplitude of IPSPs after drug application were considered to be attributable to the drug only when the effect reversed after wash out or after applications of pharmacological antagonists. Fictive swimming was elicited by a brief $(1 \mathrm{msec})$ current pulse to the flank skin delivered via copper wire on an extracellular suction electrode attached to a DS2 isolated stimulator (Digitimer, Welwyn Garden City, $\mathrm{UK})$.

Data acquisition and analysis. Data were recorded and displayed conventionally and stored on videotape using a pulse code modulator adapter (Medical Systems Corp., Greenvale, NY). Hard-copy records were made off-line using a thermal chart recorder (Graphtec, Tokyo, 
Japan). Data analysis was also performed off-line, using DataView (courtesy of Dr. William Heitler, University of St Andrews, St Andrews, UK) and the Spike 2 analysis software package (Cambridge Electronic Design, Cambridge, UK). For extracellular recordings, three consecutive episodes of fictive swimming were measured in control, drug, and wash conditions with a $30-60 \mathrm{sec}$ rest interval. For measurements within episodes of swimming, the first $500 \mathrm{msec}$ were ignored to eliminate artifacts arising from the initiating sensory stimulus, and 30 consecutive cycles of swimming activity were subsequently measured. A single average value was obtained per animal in control, drug, and wash-off conditions for motor burst durations, cycle periods, and longitudinal delays along the body (Fig. 1C2). The total duration of swimming episodes was also measured (Fig. 1C1). For intracellular experiments, measurable parameters included the amplitude of the midcycle inhibitory and on-cycle excitatory components of swimming (calculated as the peak minus the resting membrane potential for 30 cycles within three consecutive swimming episodes in each experimental condition), the frequency of IPSPs after the termination of a swimming episode (measured within $5 \mathrm{sec}$ of the end of three swimming episodes in each experimental condition), the frequency of tetrodotoxin (TTX)-resistant spontaneous IPSPs (measured in $2 \mathrm{sec}$ bins for a total of $30 \mathrm{sec}$ in each experimental condition), and the voltage deflection of the membrane potential in response to negative current pulses (measured as the resting potential minus the trough for five consecutive pulses in each experimental condition), to assess conductance changes. A single average for each intracellular parameter was calculated per animal in control, drug, and wash-off conditions. Because $S$-nitroso- $N$-acetylpenicillamine (SNAP) can depolarize motor neurons (McLean and Sillar, 2002), we were careful to return the resting membrane potential to near control levels by manually injecting current in balanced bridge mode before taking measurements. For statistical purposes, data were converted into percentage changes from the appropriate controls. These data were first arc-sine transformed to normalize the distribution and stabilize the variance of the data, and then Student's paired $t$ tests were used to assess significant changes.

In the text, data are pooled from both embryonic and larval preparations and are reported as the means \pm SEM. A separate section in the Results examines post hoc whether there were any stage-dependent differences in the reported drug effects. For figures in which the raw data are graphed (and therefore not normalized as percentage changes), either embryonic or larval data are illustrated. This is because of developmental differences in the measurable parameters of swimming (see Table 1) (Sillar et al., 1992), which, if pooled, would make the effects of each drug difficult to interpret. The appropriate stages for each graph are reported in the legends. As a result, the pooled numbers reported in the text will not correspond to those reported in the graphs.

Drug application. Drugs were bath applied by adding known quantities to the stock bottle to achieve the desired final bath concentration. SNAP was supplied by Dr. A. R. Butler (School of Chemistry, University of St Andrews) and was made fresh daily by dissolving in $0.01 \%$ dimethyl sulfoxide (DMSO). The lack of any vehicle-mediated effects using DMSO at this concentration has already been ascertained (McLean and Sillar, 2000, 2002). Arterenol (NA), phentolamine, and 2-(4carboxyphenyl)-4,4,5,5-tetramethylimidazoline-1-oxyl-3-oxide potassium salt (PTIO) were purchased from either Sigma-RBI or TocrisCookson (Bristol, UK) and made fresh daily by dissolving in distilled water. TTX was purchased from Sigma-RBI, dissolved in distilled water, and then frozen at $-20^{\circ} \mathrm{C}$ in stock solutions. Measurements were made within 10-15 min of drug application. For a recovery, measurements were made when fresh saline had recirculated for 15-20 min.

\section{Results}

There are two clear modulatory effects of NO on the fictive swimming rhythm in Xenopus tadpoles: (1) an increase in cycle periods and thus a slowing of swimming frequency and (2) a decrease in the total amount of time fictive swimming occurs, or episode duration (McLean and Sillar, 2000). Recordings from motor neurons suggest that NO achieves this by facilitating spinal glycinergic and GABAergic inhibition and by decreasing motor neuron membrane conductance (McLean and Sillar, 2002). NA has three modulatory effects on fictive swimming: (1) it also slows swimming frequency by increasing glycinergic inhibition from commissural interneurons (McDearmid et al., 1997); (2) it can increase GABA release onto motor neurons, shortening swimming episode durations (McDearmid, 1998; Fischer et al., 2001); and (3) it can decrease the longitudinal delay of motor bursts along the body by enhancing postinhibitory rebound in motor neurons (Merrywest et al., 2003). NA mediates these three effects via the activation of $\alpha$-adrenoreceptors (Fischer et al., 2001; Merrywest et al., 2002, 2003). Because of these similarities in both the effects on swimming and the underlying mechanisms, we used two pharmacological strategies to investigate the possible hierarchical relationship between NO and NA. To see whether NO was facilitating NA release, we increased exogenous levels of NO using the NO donor SNAP $(200-400 \mu \mathrm{M})$ and then blocked the targets of endogenous NA with the broad-spectrum $\alpha$-adrenoreceptor antagonist phentolamine $(40-50 \mu \mathrm{M})$. To investigate the possibility that NA may be facilitating NO production, experiments were conducted with exogenous NA $(4-8 \mu \mathrm{M})$ and the NO scavenger PTIO (100-250 $\mu \mathrm{M})$. All drugs were added sequentially.

\section{Nitrergic metamodulation of swimming frequency via noradrenaline}

In support of previous findings (McLean and Sillar, 2000, 2002), increasing extracellular concentrations of NO exogenously with SNAP (Fig. 2A1,A2,B1) increased cycle periods (and therefore slowed swimming frequency) by $\sim 20 \%(21.6 \pm 4.6 \%$; $p<0.05$; $n=19)$. There were no significant effects on burst durations $(9.5 \pm 6.2 \% ; p>0.05 ; n=19)$ or their longitudinal delay along the body $(6.6 \pm 4.6 \% ; p>0.05 ; n=19)$, also as reported previously (McLean and Sillar, 2000). The subsequent bath application of phentolamine in 10 experiments significantly counteracted the effect of NO on cycle periods by almost $10 \%(-8.2 \pm$ $3.5 \% ; p<0.05 ; n=10$ ) (Fig. 2A3,B1). This was followed by an additional reversal back to near control values after a return to fresh saline (Fig. 2A4). To determine any effects of phentolamine on endogenously released NA and also to see whether the blockade of $\alpha$-adrenoreceptors before SNAP application could occlude the effects of NO on cycle periods, we reversed the order of drug application. Phentolamine alone did not significantly affect swimming frequency $(-0.3 \pm 3.0 ; p>0.05 ; n=11)$ (Fig. 2 B2). Neither did it affect burst durations $(-3.6 \pm 2.0 \% ; p>0.05 ; n=$ $11)$ or longitudinal delays $(7.1 \pm 7.2 \% ; p>0.05 ; n=11)$. In the presence of phentolamine, the subsequent application of SNAP (Fig. 2 B2) led to a significant, yet attenuated, increase in cycle periods $(7.2 \pm 2.1 \% ; p<0.05 ; n=11)$ compared with the $\sim 20 \%$ increase measured in the absence of phentolamine (compare Fig. 2 B1). A subsequent wash returned this effect to near control values $(-5.4 \pm 4.7 \% ; p>0.05 ; n=11)$. These data strongly suggested the interpolation of a noradrenergic pathway in the effects of $\mathrm{NO}$ on fictive swimming frequency.

We next investigated whether NA facilitated NO production to control swimming frequency. When the NO scavenger PTIO was bath applied alone (Fig. 2C1,C2,D1), this led to a small, yet statistically significant decrease in cycle periods from control values $(-4.2 \pm 1.8 \% ; p<0.05 ; n=19)$. This lends additional support to our previous findings using NO synthase inhibitors, which indicated a contribution of endogenous NO to rhythm generation (McLean and Sillar, 2000). Again, we observed no significant effects on either burst durations $(-9.9 \pm 6.6 \% ; p>$ $0.05 ; n=19)$ or longitudinal delays $(-7.5 \pm 5.5 \% ; p>0.05 ; n=$ $19)$ in the presence of PTIO. However, despite the presence of 

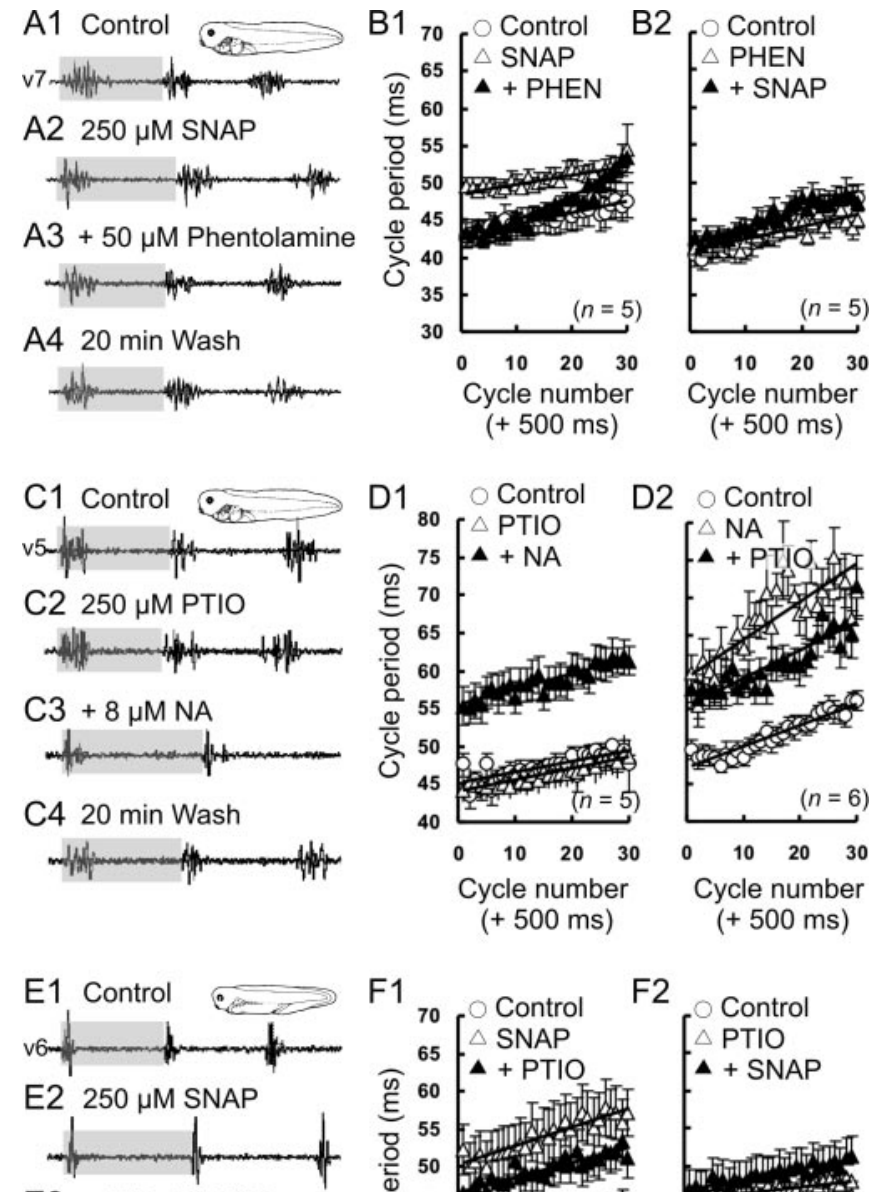

$\mathrm{E} 3+250 \mu \mathrm{MPTIO}$

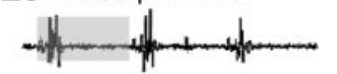

E4 20 min Wash
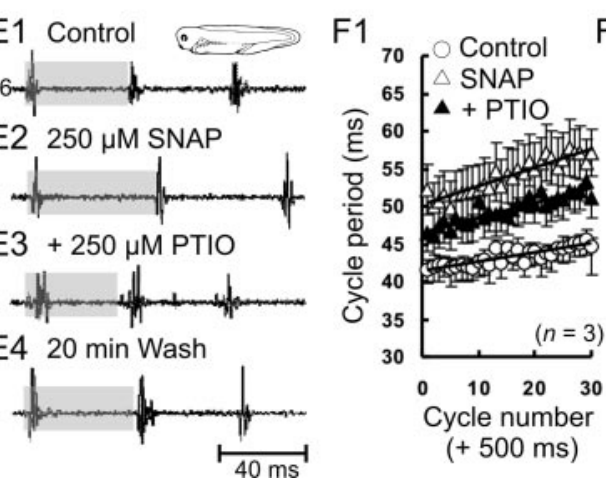

F2

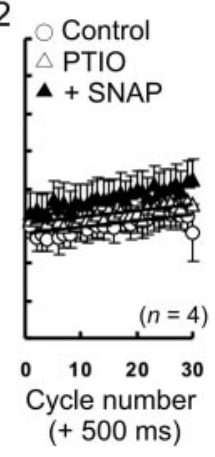

Figure 2. Nitric oxide facilitates noradrenaline release to slow swimming frequency, but not vice versa. A, Ventral root recordings from the seventh postotic cleft (v7) of a Xenopus larva (schematic inset, adapted from Nieuwkoop and Faber, 1956) illustrate that the effects of SNAP $(A 2)$ on control swimming $(A 1)$ are reversed to values by phentolamine $(A 3)$ that do not differ greatly from a recovery in fresh saline (A4). Ventral root recordings are presented as overlapping traces of consecutive cycles of motor activity (i.e., $A 1$ is actually 6 cycles of ventral root activity, not 3). Shaded gray boxes are present for illustrative purposes. B, Quantification of 30 consecutive cycles of swimming starting $500 \mathrm{msec}$ into an episode (+500 msec) from a larva (B1) and embryo (B2). The data are presented to illustrate the consistency of the resulting effects throughout the excerpt of swimming. Note that the values during phentolamine (PHEN) application eventually reach those of SNAP as a result of the concomitant effects of SNAP on episode durations. C, Ventral root recordings from the fifth postotic cleft (v5) of a Xenopus larva illustrate that the effects of PTIO (C2) on control swimming (C1) do not preclude the effects of NA (C3), which are subsequently reversed by a wash in fresh saline (C4). D, Quantification of 30 consecutive cycles of swimming 500 msec into an episode from an embryo (D1) and larva (D2). $E$, Ventral root recordings from the sixth postotic cleft (v6) of a Xenopus embryo illustrate that the effects of SNAP (E2) on control swimming (E1) are reversed to values by PTIO (E3) that do not differ greatly from a recovery in fresh saline (E4).F, Quantification of 30 consecutive cycles of swimming 500 msec into an episode (+500 msec) from embryos (F1, F2).

PTIO, subsequent application of NA in 10 experiments reversibly (Fig. 2C3,C4,D1) increased cycle periods $(22.9 \pm 4.0 \% ; p<0.05$; $n=10$ ), as reported previously (McDearmid et al., 1997). In addition, PTIO did not occlude a significant decrease in longitudinal delays $(-13.3 \pm 5.5 \% ; p<0.05 ; n=10)$, which is known to be an effect of NA (Merrywest et al., 2003). When the sequence of drug application was reversed, the increase in cycle periods induced by NA ( $38.5 \pm 6.0 \% ; p<0.05 ; n=11)$ (Fig. 2D2) was not significantly antagonized by PTIO $(-4.5 \pm 2.9 \% ; p>0.05 ; n=$ 11). Similarly, the decrease in longitudinal delays associated with NA $(-15.1 \pm 7.5 \% ; p<0.05 ; n=11)$ was not counteracted by the subsequent application of PTIO $(-4.4 \pm 2.6 \% ; p>$ $0.05 ; n=11)$.

In contrast, at similar concentrations (Fig. 2E1-E3,F1), bathapplied PTIO significantly counteracted the SNAP-mediated cycle period increase by almost 15\% $(-14.2 \pm 1.5 \%$; $p<0.05 ; n=$ $9)$, returning cycle periods to near wash values (Fig. 2E4). In addition, preapplication of PTIO significantly occluded the effects of SNAP (Fig. 2F2), limiting the increase in cycle periods to $\sim 5 \%(4.8 \pm 3.8 \% ; p>0.05 ; n=9)$ from control values. Hence, PTIO was effectively scavenging $\mathrm{NO}$ and would thus inactivate any NO produced as a result of bath-applied NA.

\section{Noradrenaline is not involved in the modulatory actions of nitric oxide on episode durations}

By monitoring the effects of different noradrenergic and nitrergic agents on swimming frequency, it appeared as though NO was facilitating NA release. If NA was also responsible for the effects of NO on episode durations, then phentolamine should successfully antagonize the effects of SNAP on this parameter. Phentolamine alone had no significantly consistent effects on episode durations $(-12.7 \pm 13.8 \% ; p>0.05 ; n=11)($ Fig. $3 A 1, A 2, B 1)$. However, in the presence of phentolamine, SNAP could still decrease episode durations (Fig. $3 A 3, B 1$ ) by as much as $60 \%$ from controls $(-61.3 \pm 8.9 \% ; p<0.05 ; n=11)$. This effect was readily reversible to near control levels after a wash in fresh saline (Fig. $3 A 4, B 1)$. Reversing the order of drug application similarly demonstrated that a $60 \%$ decrease $(-63.3 \pm 4.6 \% ; p<0.05 ; n=10)$ in episode durations by SNAP (Fig. 3B2) was not significantly reversed by phentolamine $(65.3 \pm 105.8 \% ; p>0.05 ; n=10)$. A subsequent wash in fresh saline could reverse levels to near control values (Fig. 3B2). It therefore appeared that NO was not mediating its actions on episode durations through NA.

Unlike phentolamine, preapplication of PTIO could significantly occlude the effect of SNAP on episode durations $(-14.0 \pm$ $37.0 \% ; p>0.05 ; n=9$ ) (Fig. 3C1). In addition, bath application of PTIO after SNAP significantly reversed its effects (167.2 \pm $56.7 \% ; p<0.05 ; n=9$ ) (Fig. 3C2). Notably, values measured in the presence of PTIO alone, before SNAP application, illustrated a significant increase in the average duration of swimming episodes $(165.9 \pm 58.2 \% ; p<0.05 ; n=19)$.

Like NO, NA also decreases episode durations. To rule out the interpolation of $\mathrm{NO}$ in the effects of NA on episode durations, we also monitored the effects of PTIO on this parameter after NA bath application. As observed during recordings of cycle periods within swimming episodes, PTIO did not significantly reverse or occlude the modulatory effects of NA on episode durations. For example, a $60 \%$ decrease in episode durations mediated by NA $(-59.7 \pm 10.6 \% ; p<0.05 ; n=11)$ was not reversed by the subsequent application of PTIO $(39.2 \pm 39.8 \%$; $p>0.05 ; n=$ 11). Similarly, in the presence of PTIO, NA was still able to decrease episode durations by as much as $30 \%(-31.5 \pm 12.0 \%$; $p<0.05 ; n=10)$. This markedly contrasts the potent antagonistic effects of PTIO on SNAP. Collectively, these data suggest that $\mathrm{NO}$ was primarily modulating spinal glycinergic inhibition indirectly through a noradrenergic pathway, while directly facilitating GABAergic inhibition in the spinal cord. 

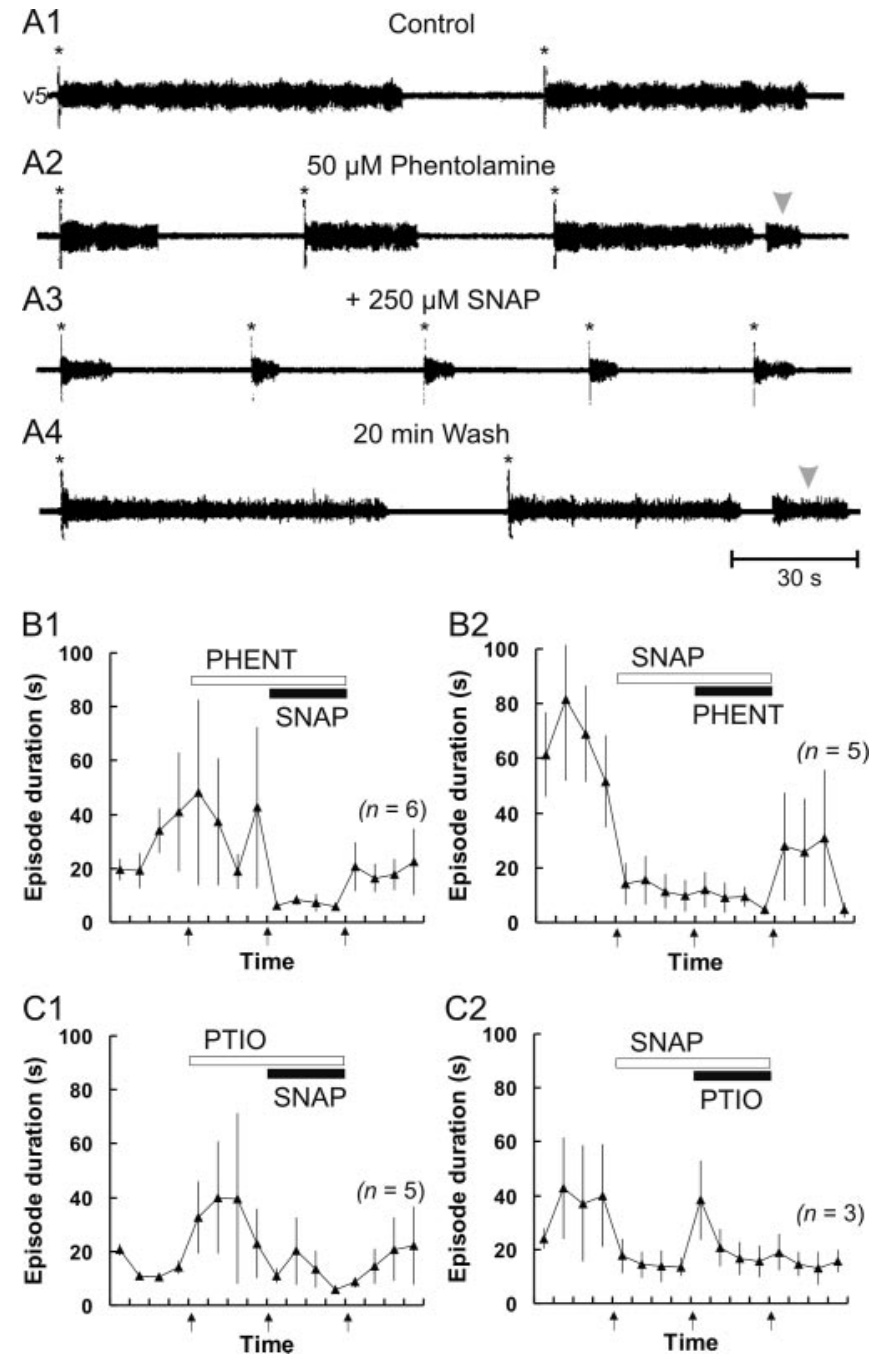

Figure 3. Nitric oxide decreases episode durations despite $\alpha$-adrenoreceptor antagonism. $A$, Ventral root recording from the fifth postotic cleft (v5) of a Xenopus embryo illustrates whole swimming episodes elicited by electrical stimulation $\left(^{*}\right)$ at 30 sec intervals after the end of the preceding episode. Clearly, in the presence of phentolamine (PHENT) (A2), SNAP can still decrease episode durations (A3) from control values (A1), an effect which is reversible (A4). Note spontaneous swimming episodes at gray arrowheads $(\boldsymbol{\nabla}) . B, C$, The mean \pm SEM response $(\mathbf{\Delta})$ illustrates the effects of a drug (open bars) and the subsequent effects after addition of a different drug (closed bars). The sample interval between open circles is $30 \mathrm{sec}$, except for at the black arrows $(\uparrow)$, which designate an interval of $10-20$ min. Note that $B 1, B 2$, and $C 1$ are from larvae, whereas $C 2$ is from embryos.

\section{Developmental considerations}

Our primary goal was to ascertain whether NO might control NA release to modulate locomotion. However, we were aware that the relative contribution of each amine could change as the animal develops, which would shed additional light on the mechanisms of nitrergic modulation. For example, noradrenergic spinal innervation is already present by stage 37/38 (González et al., 1994), whereas the 5-HT pathway does not fully innervate the spinal cord until stage 41 (van Mier et al., 1986). In addition, although $\mathrm{NO}$ appears to be present in close proximity to the locus ceruleus and GABAergic mid-hindbrain reticulospinal region by stage $37 / 38$, it does not localize near the serotonergic raphe region until stage 41 (McLean and Sillar, 2001). Therefore, we ensured that there were sufficient numbers of experiments with both embryos and larvae to enable a post hoc comparison of the pharmacological effects of the various drugs applied. In the interests of brevity, these have been tabulated (Table 1). Consistent with the primary involvement of noradrenergic pathways in the nitrergic metamodulation of swimming frequency, SNAP could significantly increase cycle periods in both embryos and larvae (Table 1, row 2, columns 2 and 6). This effect was readily antagonized at both developmental stages by phentolamine (Table 1, row 4, columns 2 and 6). In support of a direct modulation of GABA release by NO, SNAP was also able to significantly decrease episode durations at both developmental stages (Table 1, row 2, columns 4 and 8), which was similarly unaffected by the application of phentolamine (Table 1, row 4, columns 4 and 8). One major developmental difference was the effect of manipulating endogenous levels of NO using PTIO. This was more successful in larvae than in embryos (Table 1, row 5, columns 2, 4, 6, and 8), suggesting that the endogenous mechanisms of nitrergic metamodulation may not yet be active at earlier stages. Given the differences in the consistency of endogenous effects between embryos and larvae, we decided to limit our intracellular investigation to motor neurons from larvae (stages 41-42). However, sharp microelectrode recordings from larval motor neurons are exceptionally difficult, so the sample size of the experiments that follow is low relative to the extracellular data set $(n=10)$.

\section{Synaptic mechanisms of nitrergic metamodulation}

The data from our extracellular experiments suggested that NO was directly potentiating GABAergic inhibition but indirectly modulating glycinergic inhibition via NA. Therefore, we expected during recordings from presumed motor neurons that there would be a selective potentiation of GABAergic over glycinergic inhibition by SNAP in the presence of phentolamine. It has been shown previously that GABA inhibitory IPSPs coincide with the termination of swimming episodes (Reith and Sillar, 1999), whereas glycinergic IPSPs coincide with the midcycle component of swimming (Soffe, 1987). Bath application of phentolamine alone did not appear to have any significant effect on the frequency of IPSPs after the end of each episode (Fig. 4A1,A2,C1). Nor were there any significant effects of the amplitude of midcycle inhibition (Fig. 4 B1,B2,D1) or on-cycle excitation $(-4.1 \pm$ $2.4 \% ; p>0.05 ; n=3$ ) (Fig. $4 B 1, B 2$ ), indicating little or no endogenous activation of adrenoreceptors. The subsequent bath application of SNAP significantly increased the occurrence of IPSPs that immediately followed swimming episodes compared with those recorded in control conditions (Fig. 4A3,C1). This effect was readily reversible (Fig. $4 A 4, C 1$ ). The increase in the occurrence of terminating IPSPs was also associated with a reversible decrease in episode durations (Fig. 4C2). As we also predicted, the addition of SNAP in the presence of phentolamine failed to change the amplitude of midcycle inhibition from controls (Fig. 4B3,D1). This markedly contrasts with the normal response to SNAP, in which midcycle inhibition increased by as much as 30\% (McLean and Sillar, 2002). We also reported previously that SNAP has no effect on the amplitude of on-cycle excitation (McLean and Sillar, 2002). Here, in the presence of phentolamine, SNAP similarly failed to affect the amplitude of on-cycle excitation $(-1.9 \pm 4.1 \% ; p>0.05 ; n=3)$ (Fig. 4 B3). Consistent with this lack of effect on midcycle inhibition and on-cycle excitation, there was no significant change in cycle periods observed with SNAP in these intracellular experiments in which phentolamine had been preapplied (Fig. 4D2). A wash in fresh saline also yielded no significant change in either midcycle or on-cycle amplitude or cycle periods (Fig. 434,D2). Importantly, these data corroborate our extracellular experiments and suggest that NO is indeed shortening episode durations directly 
Table 1. Developmental comparison of nitrergic metamodulation of cycle periods and episode durations

\begin{tabular}{|c|c|c|c|c|c|c|c|c|}
\hline \multirow[b]{2}{*}{ Drug (action) } & \multicolumn{4}{|c|}{ Embryonic stage $37 / 38$} & \multicolumn{4}{|l|}{ Larval stage 42} \\
\hline & CP (in milliseconds) & Percentage difference & $E D$ (in seconds) & Percentage difference & CP (in milliseconds) & Percentage Difference & $\mathrm{ED}$ (in seconds) & Percentage difference \\
\hline None (control) & $46.4 \pm 1.0(27)$ & $0.0 \pm 0.0(27)$ & $52.3 \pm 6.1(27)$ & $0.0 \pm 0.0(27)$ & $52.2 \pm 1.2(33)$ & $0.0 \pm 0.0(33)$ & $30.2 \pm 4.6(33)$ & $0.0 \pm 0.0(33)$ \\
\hline SNAP (+N0) & $56.1 \pm 2.5(8)$ & $22.9 \pm 6.3(8)^{*}$ & $11.4 \pm 1.7(8)$ & $-67.2 \pm 6.2(8)^{*}$ & $64.8 \pm 3.8(11)$ & $20.3 \pm 6.7(11)^{*}$ & $13.1 \pm 4.5(11)$ & $-60.0 \pm 6.9(11)^{*}$ \\
\hline Plus PTIO (-NO) & $44.8 \pm 2.5(3)$ & $-10.9 \pm 3.4(3)^{* *}$ & $22.8 \pm 8.3(3)$ & $251.0 \pm 158.4(3)$ & $53.6 \pm 3.6(6)$ & $-15.9 \pm 1.1(6)^{* *}$ & $21.4 \pm 1.9(6)$ & $83.3 \pm 28.5(6)^{* *}$ \\
\hline Plus PHEN (-NA) & $46.7 \pm 1.9(5)$ & $-7.6 \pm 0.7(5)^{* *}$ & $11.9 \pm 5.0(5)$ & $-25.3 \pm 16.8(5)$ & $54.3 \pm 5.1(5)$ & $-8.8 \pm 1.3(5)^{* *}$ & $8.9 \pm 3.5(5)$ & $65.3 \pm 105.8(5)$ \\
\hline PTIO (-NO) & $46.1 \pm 2.7(9)$ & $-0.1 \pm 2.2(9)$ & $61.7 \pm 11.1(9)$ & $79.5 \pm 65(9)$ & $48.8 \pm 1.9(10)$ & $-7.9 \pm 2.3(10)^{*}$ & $71.9 \pm 8.7(10)$ & $243.6 \pm 90.0(10)^{*}$ \\
\hline Plus SNAP (+N0) & $48.8 \pm 4.2(4)$ & $7.4 \pm 1.8(4)^{*}$ & $27.9 \pm 13.5(4)$ & $3.1 \pm 62.7(4)$ & $50.2 \pm 4.7(5)$ & $2.8 \pm 7.1(5)$ & $16.8 \pm 7.1(5)$ & $-21.6 \pm 15.5(5)$ \\
\hline Plus NA (+NA) & $59.1 \pm 2.9(5)$ & $25.9 \pm 5.6(5)^{*}$ & $21.1 \pm 5.1(5)$ & $-53.2 \pm 8.3(5)^{*}$ & $69.6 \pm 5.8(5)$ & $19.9 \pm 5.9(5)^{*}$ & $24.2 \pm 3.1(5)$ & $-9.7 \pm 18.5(5)$ \\
\hline$N A(+N A)$ & $62.2 \pm 3.7(5)$ & $34.3 \pm 7.5(5)^{*}$ & $21.5 \pm 9.4(5)$ & $-33.5 \pm 17.5(5)^{*}$ & $68.3 \pm 2.1(6)$ & $41.9 \pm 9.4(6)^{*}$ & $9.7 \pm 2.7(6)$ & $-46.1 \pm 13.9(6)^{*}$ \\
\hline Plus PTIO (-N0) & $58.0 \pm 2.7(5)$ & $-6.4 \pm 3.6(5)$ & $34.2 \pm 6.8(5)$ & $131.6 \pm 57.9(5)$ & $65.9 \pm 2.8(6)$ & $-2.9 \pm 4.5(6)$ & $19.9 \pm 13.8(6)$ & $-1.8 \pm 20.6(6)$ \\
\hline PHEN (-NA) & $50.0 \pm 2.2(5)$ & $6.4 \pm 1.6(5)^{*}$ & $53.5 \pm 21.6(5)$ & $-25.9 \pm 18.8(5)$ & $43.0 \pm 1.7(6)$ & $-5.6 \pm 4.3(6)$ & $37.7 \pm 21.6(6)$ & $-1.7 \pm 20.3(6)$ \\
\hline Plus SNAP $(+N 0)$ & $52.2 \pm 3.8(5)$ & $10.4 \pm 3.2(5)^{*}$ & $20.3 \pm 12.9(5)$ & $-75.0 \pm 11.8(5)^{*}$ & $47.3 \pm 1.5(6)$ & $3.4 \pm 3.2(6)$ & $7.1 \pm 1.4(6)$ & $-61.7 \pm 13.9(6)^{*}$ \\
\hline
\end{tabular}

Data are expressed as means \pm SEM with the $n$ in parentheses. Statistical evaluations between animals were performed on percentage values as detailed in Materials and Methods. Note that percentage differences are from the corresponding control values and not from the control values given in the top column. This is present only for the purposes of comparison. ${ }^{*} p<0.05$ significance determined by Student's paired $t$ test with corresponding control values. ${ }^{* *} p<$ 0.05 significance determined by Student's paired $t$ test with previous drug values. CP, Cycle period; ED, episode duration; PHEN, phentolamine.

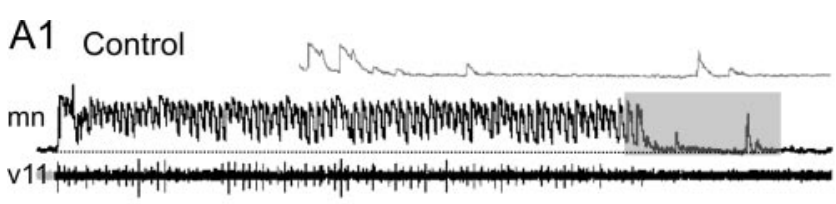

A2 $50 \mu \mathrm{M}$ Phentolamine

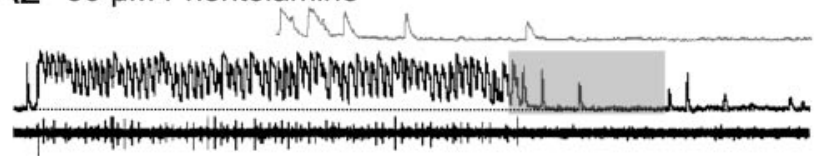

A3 $+200 \mu \mathrm{M}$ SNAP

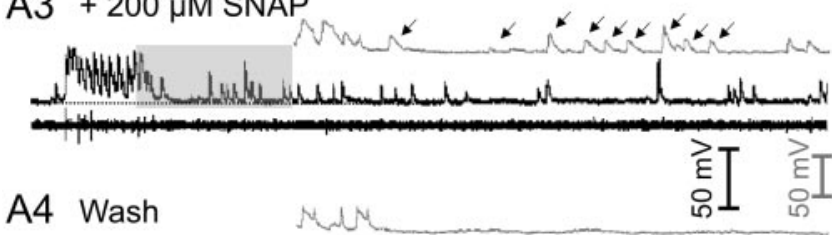

A4 Wash

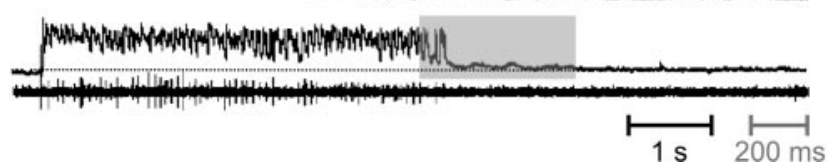

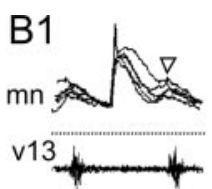

B2

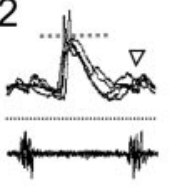

B3 ……
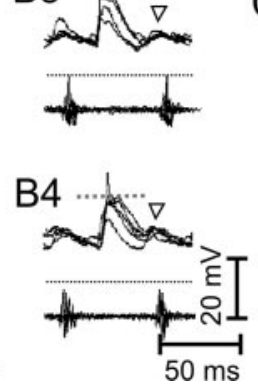

C1

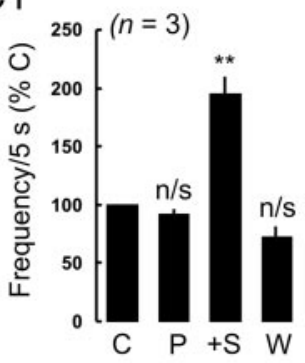

D1

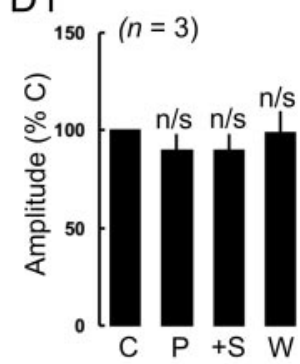

C2

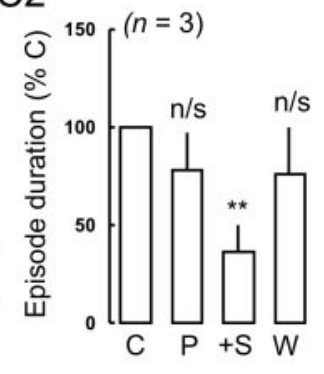

D2

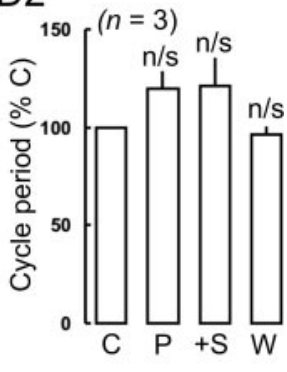

Figure 4. $\quad \alpha$-Adrenoreceptor antagonism cannot occlude the increase in inhibition by nitric oxide that can terminate episodes of swimming but occludes the effect of nitric oxide on midcycle inhibition. $A$, Simultaneous recordings from a motor neuron $(\mathrm{mn})$ and the ventral root at the 11th postotic cleft (v11) of a Xenopus larva in control conditions (A1), the presence of phentolamine (A2), SNAP (A3), and a wash in fresh saline (A4). Excerpts on a faster time scale (gray inset) are taken from the region in the shaded gray box and more clearly illustrate the increase in inhibition at the termination of the swimming episode in the presence of SNAP $(\downarrow)$. The appropriate scale bars are coded according to the shade used. The resting membrane potential is demarcated by a dotted line and is $-59,-59,-60$, and $-55 \mathrm{mV}$ for $A 1-A 4$, respectively. $B$, Simultaneous recordings from a different preparation illustrated as five consecutive overlapping traces starting $500 \mathrm{msec}$ into an episode in control conditions (B1), the presence of $50 \mu \mathrm{m}$ phentolamine (B2), $500 \mu \mathrm{m} \mathrm{SNAP} \mathrm{(B3),} \mathrm{and} \mathrm{a} \mathrm{wash} \mathrm{in} \mathrm{fresh} \mathrm{saline} \mathrm{(B4).} \mathrm{The} \mathrm{resting} \mathrm{membrane} \mathrm{potential} \mathrm{is} \mathrm{noted} \mathrm{by} \mathrm{a} \mathrm{dotted} \mathrm{line} \mathrm{and}$ is $-60,-60,-59$, and $-55 \mathrm{mV}$ for $B 1-B 4$, respectively. A gray dashed line representing the control amplitude is present for comparisons between conditions. Note that neither midcycle inhibition nor on-cycle excitation are affected by either phentolamine or SNAP $(\nabla)$. C, Quantification of the effects on the frequency of terminating IPSPs within 5 sec of the end of an episode (C1) and episode duration (C2), expressed as a percentage change from the appropriate controls (\% C). D, Quantification of the effects on midcycle inhibition amplitude (D1) and cycle period (D2), expressed as a percentage change from the appropriate controls. C, Control; $\mathrm{P}$, phentolamine; S, SNAP; W, wash; $\mathrm{n} / \mathrm{s}$, not significant at $p<0.05$; ${ }^{*}$ significant at $p<0.05$.

through the potentiation of GABA release but slowing swimming frequency by increasing inhibition at midcycle via NA release.

To more clearly illustrate that NO could selectively potentiate GABA release presynaptically in the face of adrenoreceptor antagonism, we also conducted experiments in the presence of the sodium channel blocker TTX. Under these conditions, spontaneous IPSPs are readily identifiable, with shorter-duration $(\leq 90$ $\mathrm{msec}$ ) IPSPs reflecting glycine release and longer-duration $(\geq 90$ msec) IPSPs reflecting GABA release (Fig. 5A1) (Reith and Sillar, 1997; McLean and Sillar, 2002). Spontaneous EPSPs do not contribute significantly to the spontaneous PSPs observed at these stages of development (Reith and Sillar, 1997; McLean and Sillar, 2002). Consistent with its lack of effect on evoked release, phen- tolamine alone did not change the frequency of spontaneous glycine or GABA release (Fig. 5A2, $B, C$ ) from control values (glycine, $1.9 \pm 0.4 / 2 \mathrm{sec}$; GABA, $0.5 \pm 0.1 / 2 \mathrm{sec} ; n=3$ ). However, the subsequent bath application of SNAP selectively potentiated the number of GABAergic IPSPs (glycine, $2.6 \pm 0.7 / 2 \mathrm{sec}$; GABA, $2.1 \pm 0.2 / 2 \mathrm{sec} ; n=3$ ) (Fig. $5 A 3, B, C$ ). This contrasts the normal potentiation of both glycinergic and GABAergic IPSPs by SNAP [cf. McLean and Sillar (2002), their Figs. 5 and 6]. This effect was readily reversible (glycine, $1.7 \pm 0.2 / 2 \mathrm{sec}$; GABA, $0.7 \pm 0.2 / 2 \mathrm{sec}$; $n=3$ ) (Fig. 5A4,C).

It is worth noting that in the presence of phentolamine, SNAP still consistently depolarized the motor neuron resting membrane potential by $5-10 \mathrm{mV}(5.5 \pm 2.2 \mathrm{mV} ; n=3)$. Phentol- 

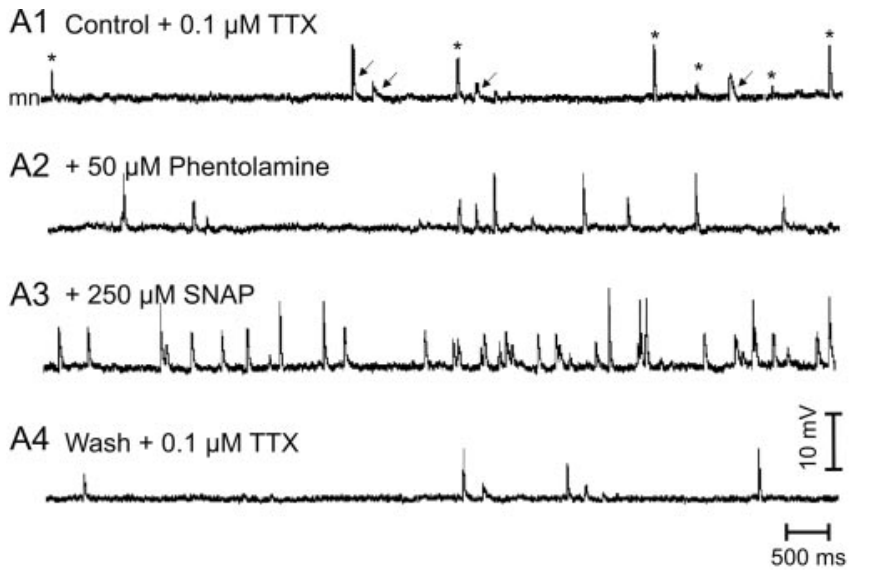

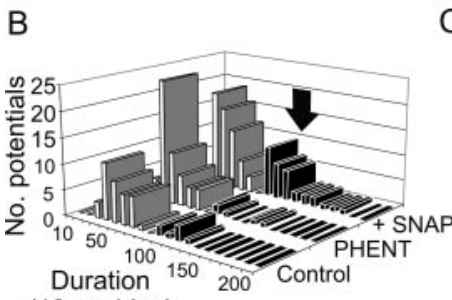

(10 ms bins)

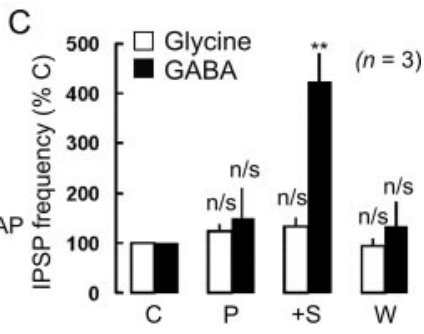

Figure 5. Nitric oxide potentiates only GABA release when $\alpha$-adrenoreceptors are antagonized. $A$, Intracellular recording from a motor neuron $(\mathrm{mn})$ in the presence of TTX illustrates how easily glycinergic $\left(^{*}\right)$ and GABAergic $(\downarrow)$ IPSPs can be distinguished based on their durations in control conditions (A1), in the presence of phentolamine (A2), SNAP $(A 3)$, and after a wash in fresh saline (A4). Resting membrane potentials are $-62,-65,-65$, and $-64 \mathrm{mV}$ for $A 1-A 4$, respectively. $B$, Three-dimensional histogram illustrates the data from one experiment in which the total number of IPSPs in a 30 sec period in each condition are separated according on their duration. Note, at black arrow, that SNAP only potentiates longer duration IPSPs in the presence of phentolamine (PHENT). C, Quantification of glycinergic ( $\square$ ) and GABAergic ( $\square$ ) IPSPs for all three experiments are reported as percentage changes from control values $(\% \mathrm{C})$. Note the significant increase in the percentage of only GABAergic IPSPs, which contrasts the normal effects of SNAP on both glycinergic and GABAergic IPSPs in the absence of phentolamine (McLean and Sillar, 2002). C, Control; P, phentolamine; S, SNAP; W, wash; $n / s$, not significant at $p<0.05 ;{ }^{* *}$ significant at $p<0.05$.

amine, on the other hand, had no observable effects on resting membrane potential $(-0.4 \pm 0.8 \mathrm{mV} ; n=3)$. We have previously hypothesized that this depolarization induced by SNAP is the result of a direct blockade of a passive $\mathrm{K}^{+}$-conductance in motor neurons, leading to a concomitant decrease in membrane conductance (McLean and Sillar, 2002). During all our measurements, we were careful to manually return the resting membrane potential to near control levels. In the presence of TTX, this resulted in a significant $\sim 40 \%$ increase $(40.9 \pm 8.1 \%$; $n=3$; paired $t$ test; $p<0.05$ ) in the amplitude of spontaneous IPSPs (Fig. $6 B$ ) compared with amplitudes measured at a similar membrane potential to control conditions $(7.6 \pm 2.1 \mathrm{mV} ; n=3)$. This effect was readily reversible to near control values $(7.1 \pm 1.5 \mathrm{mV} ; n=3)$ and is consistent with a decrease in membrane conductance. To test this further, we injected negative current pulses and noted the resulting deflections in membrane potential. As expected, in the presence of phentolamine, SNAP was still able to decrease membrane conductance (Fig. 6A), which was quantified as a significant, yet reversible, $\sim 20 \%$ increase in membrane resistance (Fig. $6 C$ ). This supports our previous findings (McLean and Sillar, 2002) and suggests that in addition to the indirect effects of NO on glycinergic inhibition via NA and the direct effects on GABAergic inhibition, NO is directly affecting motor neuron membrane properties.

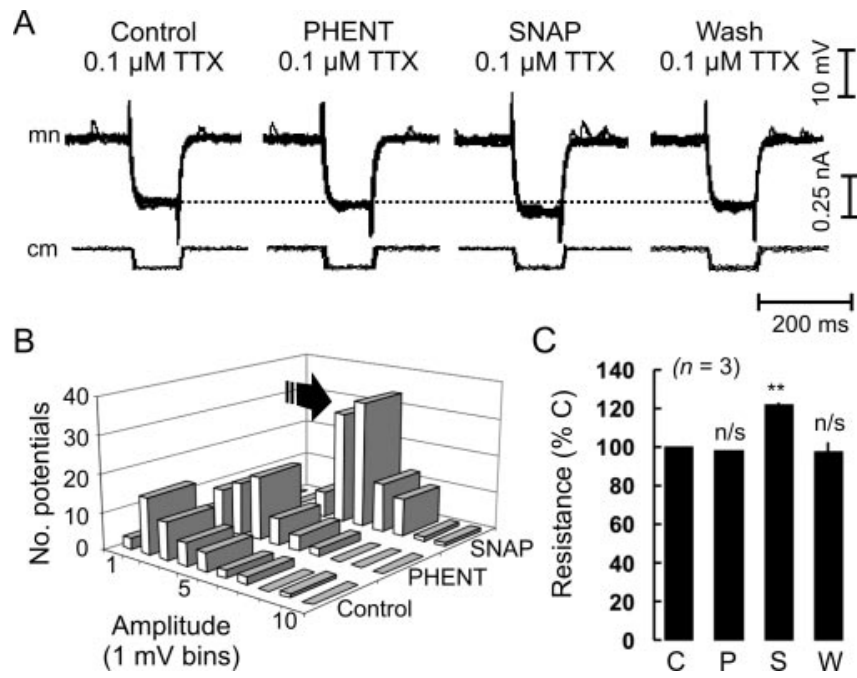

Figure 6. Nitric oxide still has direct effects on motor neuron membrane properties after blockade of adrenoreceptors. $A$, Intracellular recording from a motor neuron $(\mathrm{mn})$ in the presence of TTX showing voltage deflections in membrane potential after injection of negative current pulses [current monitor $(\mathrm{cm})$ ], in control conditions, the presence of $50 \mu \mathrm{m}$ phentolamine, $200 \mu \mathrm{M}$ SNAP, and a recover wash, illustrated as five consecutive overlapping traces. A horizontal dotted line is present for comparative purposes and illustrates the reversible effect of SNAP on membrane conductance-resistance. Resting membrane potentials are $-68,-70$, -68 , and $-66 \mathrm{mV}$ for control, PHENT, SNAP, and wash, respectively. $B$, Three-dimensional histogram illustrates the data from one experiment in which the total number of IPSP s within 30 sec in each condition are separated according on their amplitude. Note, at arrow, that SNAP shifts the amplitude distribution to the right, indicating an increase in the average amplitude of spontaneous IPSPs as a result of an increase in membrane resistance. C, Quantification of the increase in voltage deflection in response to a constant-current pulse (increase in membrane resistance), expressed as a proportion of the corresponding controls (\% C). C, Control; P, PHENT, phentolamine; S, SNAP; W, wash; $\mathrm{n} / \mathrm{s}$, not significant at $p<0.05$; ${ }^{* *}$ significant at $p<0.05$.

Finally, the acute time course of PTIO provided us with the opportunity to confirm the synaptic mechanisms underlying the endogenous actions of $\mathrm{NO}$ on swimming activity. We were previously prevented from doing so because of the long time of onset using NO synthase inhibitors $(\sim 1-2 \mathrm{hr}$ ) (McLean and Sillar, 2000) and the difficulty in holding stable recordings for such a prolonged period. Given the more rapid effects of PTIO alone on fictive swimming, as described above, and the known synaptic effects of SNAP (McLean and Sillar, 2002), we predicted that PTIO would decrease the occurrence of GABAergic IPSPs after swimming episodes and the amplitude of glycinergic inhibition at midcycle. Indeed, bath application of PTIO reversibly decreased the occurrence of IPSPs that follow swimming episodes (Fig. 7A1-A3,C1). This was also associated with a noticeable increase in the duration of episodes (Fig. 7A1-A3,C2). Although this proved insignificant after statistical analysis $(178.9 \pm 38.8 \%$; $p=0.09 ; n=4)$, the effect of PTIO on episode durations from the larger extracellular data set was significant (see above). Also as we predicted, in the presence of PTIO, midcycle IPSP amplitudes reversibly decreased (Fig. 7B1-B3,D1), as did cycle periods (Fig. $7 D 2$ ), again corroborating our extracellular experiments. As we observed using SNAP, we did not note any significant effects of PTIO on the amplitude of on-cycle excitation $(2.7 \pm 6.8 \% ; p>$ $0.05 ; n=4)$.

To more accurately assess the effects of PTIO on glycinergic and GABAergic inhibition, we monitored its effects on spontaneously occurring IPSPs during interepisode quiescent periods (Fig. 8A1). Consistent with its effects on evoked transmitter release during swimming, PTIO application led to a decrease in the 

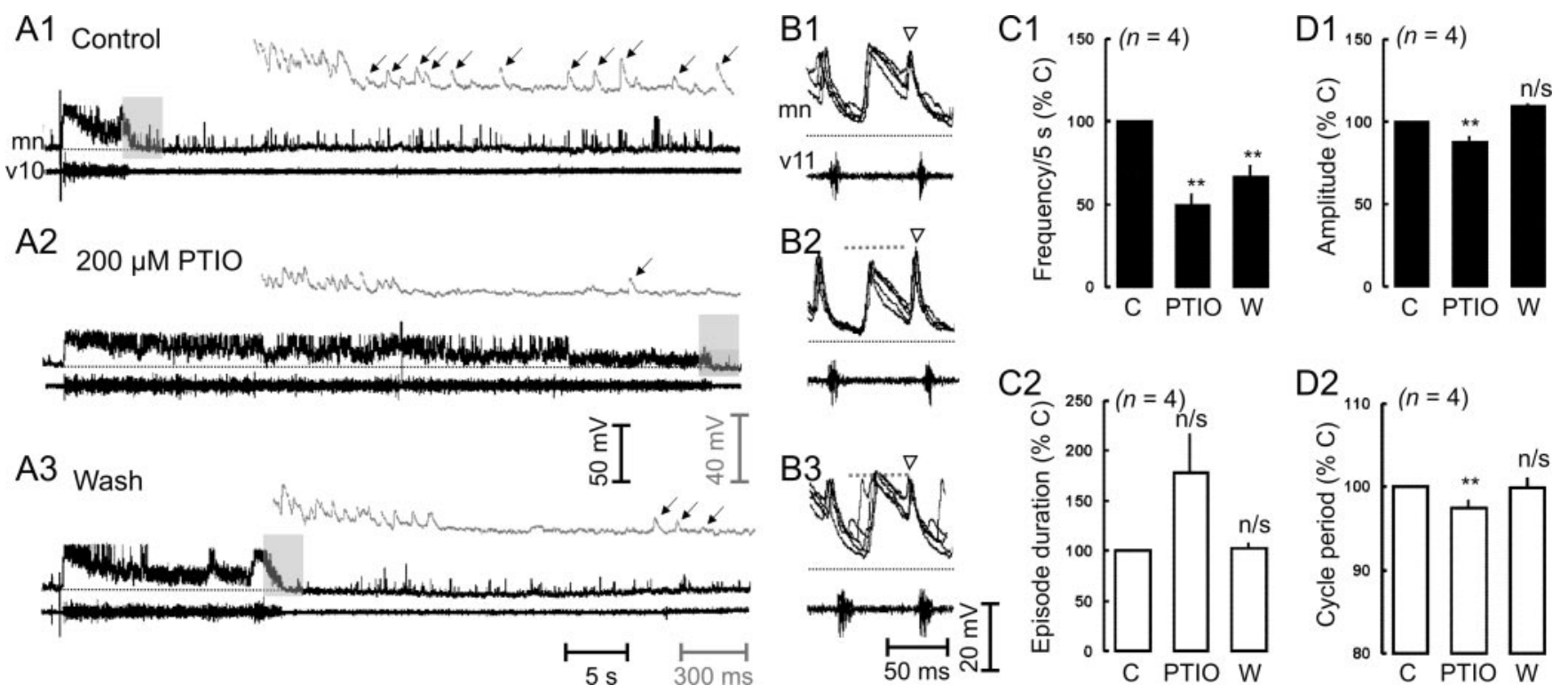

Figure 7. Endogenous nitrergic metamodulation of glycinergic and GABAergic inhibition during swimming. $A$, Simultaneous recordings from a motor neuron $(\mathrm{mn})$ and the ventral root at the 10 th postotic cleft (v10) of a Xenopus larva in control conditions ( $A 1$ ), the presence of PTIO (A2), and a wash in fresh saline ( $A 3$ ). Excerpts on a faster time scale (gray inset) are taken from the region in the shaded gray box and more clearly illustrate the decrease in inhibition at the termination of the swimming episode in the presence of PTIO ( $\downarrow$ ), which is partially reversible. The appropriate scale bars are coded according to the shade used. The resting membrane potential is demarcated by a dotted line and is $-62,-66$, and $-64 \mathrm{mV}$ for $A 1-A 3$, respectively. $B$, Simultaneous recordings from a different preparation illustrated as five consecutive overlapping traces starting $500 \mathrm{msec}$ into an episode in control conditions (B1), the presence of $200 \mu \mathrm{m}$ PTIO (B2), and a wash in fresh saline (B3). The resting membrane potential is noted by a dotted line and is $-61,-64$, and $-60 \mathrm{mV}$ for $B 1-B 3$, respectively. A gray dashed line representing the control amplitude is present for comparisons between conditions. Note that PTIO does not affect on-cycle excitation $(\nabla)$. C, Quantification of the effects on the frequency of terminating IPSPs within 5 sec of the end of an episode (C1) and episode duration (C2), expressed as a percentage change from the appropriate controls (\% C). D, Quantification of the effects on midcycle inhibition amplitude (D1) and cycle period (D2), expressed as a percentage change from the appropriate controls. C, Control; W, wash; $\mathrm{n} / \mathrm{s}$, not significant at $p<0.05$; ** significant at $p<0.05$.

occurrence of spontaneous IPSPs (Fig. 8A2). Separating IPSPs according to their durations clearly demonstrated that PTIO consistently decreased the occurrence of both glycinergic (1.3 \pm $0.1 / 2 \sec ; n=4)$ and GABAergic $(0.1 \pm 0.01 / 2 \mathrm{sec} ; n=4)$ IPSPs (Fig. $8 B, C$ ), when compared with control values (glycine, $2.6 \pm$ $0.5 / 2 \mathrm{sec}$; GABA, $0.3 \pm 0.06 / 2 \mathrm{sec} ; n=4$ ). Again, this effect was partially reversible by a wash in fresh saline (Fig. $8 A 3, B, C$ ). Collectively, these data support our previous findings with $\mathrm{NO}$ synthase inhibitors and suggest that endogenous NO metamodulates the locomotor network for swimming by promoting the glycinergic and GABAergic inhibition that impinges on motor neurons.

\section{Discussion}

Our results suggest both serial and parallel actions of NO and NA on spinal network inhibition. Both NO and NA have been reported to have direct effects on motor neuron membrane properties (McLean and Sillar, 2002; Merrywest et al., 2003). We hypothesized that the direct conductance decrease in motor neurons mediated by $\mathrm{NO}$ acts to selectively potentiate inhibition as a result of the concomitant effect of NO on glycinergic and GABAergic IPSP frequency (McLean and Sillar, 2002). Our current experiments support a direct effect of $\mathrm{NO}$ on motor neurons, because the conductance decrease mediated by SNAP was phentolamine resistant. A direct $\alpha_{1}$-adrenoreceptor-mediated potentiation of postinhibitory rebound firing in motor neurons is thought to contribute to the effect of NA on longitudinal delays (Merrywest et al., 2003). Our experiments demonstrate that this is also a direct effect of NA, because the modulation of longitudinal delays by NA persisted in the presence of PTIO. Both NO and NA also have been reported to have direct effects on GABAergic inhibition, thus shortening episode durations (McLean and Sillar, 2002; Merrywest et al., 2002). Again, our experiments support this conclusion, because the effects of SNAP on GABAergic IPSP frequency and episode durations persisted in the presence of phentolamine. Similarly, NA could still decrease episode durations despite NO scavenging with PTIO. Finally, both NO and NA have been reported to facilitate glycinergic inhibition, thus slowing swimming frequency (McDearmid et al., 1997; McLean and Sillar, 2002). Whereas our experiments demonstrated that NA was directly increasing glycine release, as illustrated by its PTIO-resistant effects on swimming frequency, it was clear that $\mathrm{NO}$ was not acting directly on glycinergic pathways. This was evidenced by the antagonistic effects of phentolamine on the modulation of swimming frequency and glycinergic inhibition by SNAP.

\section{Site of metamodulatory interaction}

The present study has not addressed the possible sites of interaction between NO, NA, GABA, and glycine. The simplest proposal is that the main site of interaction is the spinal cord itself (Fig. 9). This is based on the fact that the potentiating effect of SNAP on glycinergic and GABAergic IPSPs persists in the presence of TTX (McLean and Sillar, 2002). In addition, NO also has direct TTXresistant effects on spinal motor neurons (McLean and Sillar, 2002). However, this evidence alone does not rule out possible interactions with descending brainstem systems (NA in the locus ceruleus; GABA from the mid-hindbrain reticulospinal system) (Fig. 9), which we presume also occur. Although future spinalization experiments could shed light on this issue, it seems unlikely that we will be able to segregate NO-NA interactions quite so neatly, because the direct spinal actions probably contribute significantly to the effects of $\mathrm{NO}$ on fictive locomotion. In addition, although it is possible to record swimming after spinalization, a proportion of the spinal pattern generator extends into the rostral hindbrain (Roberts and Alford, 1986) and overlaps with nitrergic neurons we have described previously (McLean and Sil- 


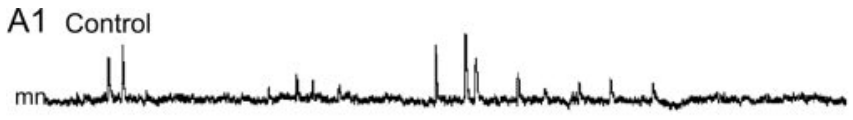

A2 $100 \mu \mathrm{M}$ PTIO

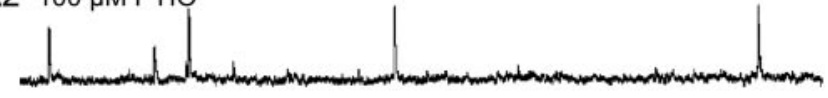

A3 Wash
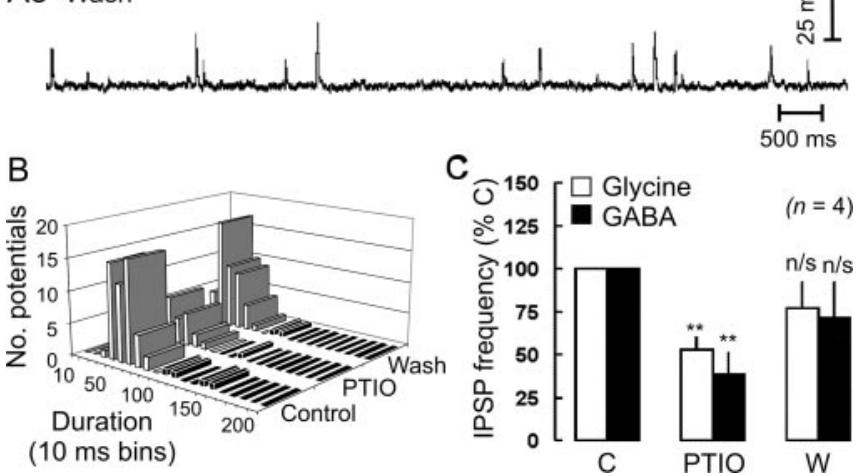

Figure 8. Scavenging endogenous nitric oxide also decreases spontaneous glycinergic and GABAergic IPSPs during interepisode quiescent periods. $A$, Intracellular recording from a motor neuron (mn) in control conditions (A1), in the presence of PTIO (A2), and after a wash in fresh saline (A3) illustrates the reversible effect of PTIO on spontaneous IPSPs. Resting membrane potentials are $-70,-71$, and $-69 \mathrm{mV}$ for $A 1-A 3$, respectively. $B$, Three-dimensional histogram illustrates the data from one experiment, in which the total number of IPSPs in a $30 \mathrm{sec}$ period in each condition are separated according on their duration. Note that PTIO decreases both short duration glycinergic IPSPs and longer duration GABAergic IPSPs. C, Quantification of glycinergic $(\square)$ and GABAergic $(\square)$ IPSPs for all four experiments are reported as percentage changes from control values $(\% \mathrm{C})$. Note the significant decrease in the percentage of both glycinergic and GABAergic IPSPs. C, Control; W, wash; $\mathrm{n} / \mathrm{s}$, not significant at $p<0.05$; **significant at $p<0.05$.

lar, 2000, 2001). After removal of all of the nitrergic systems, the remaining spinal circuitry will likely have much-reduced rhythm-generating capability, making it difficult to analyze and interpret data compared with intact animals (McLean and Sillar, 2003). Finally, the process of spinalization and the cutting of descending axons will lead to the release of multiple transmitters and modulators onto the spinal networks, which may change the way the network responds to subsequent manipulations (cf. Thoby-Brisson and Simmers, 1998). Thus, any future attempts to identify the loci for NO interactions will have to keep these caveats in mind.

\section{Modulation of descending systems by nitric oxide}

Anatomical evidence regarding the ontogenetic expression of NO synthase indicates that it localizes first in the region of GABAergic and noradrenergic populations, then near the raphe and, by stage 46, in the spinal cord (McLean and Sillar, 2001). Clearly, this suggests that the endogenous actions of NO may not appear until later larval life. However, the staggered appearance of NOgenerating capacity does not preclude the ability of exogenous NO to modulate target neurons (in the brainstem or spinal cord), which may express subcellular NO targets ahead of NO production. This is supported by our manipulations of endogenous levels of NO using the NO scavenger PTIO, which were more reliably effective in larval preparations.

Related to this issue, a recent study has investigated the potential involvement of NO in the mid-hindbrain reticulospinalmediated stopping response in Xenopus embryos (Lambert et al., 2004). At this early stage in development, they failed to find a

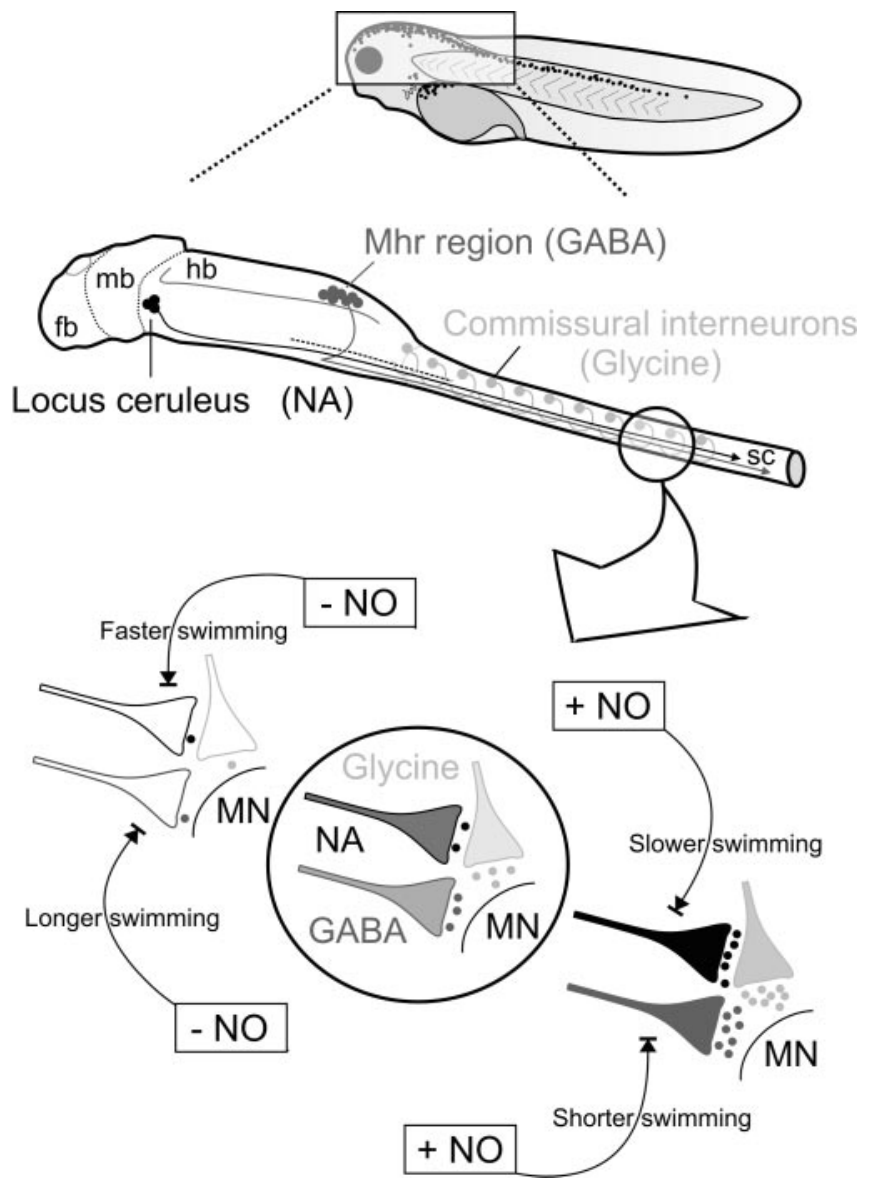

Figure 9. Summary diagram illustrating the concerted metamodulation of spinal inhibition by nitric oxide. At larval stage 42 (top schematic), the major subdivisions of the CNS (middle schematic) are recognizable, including the forebrain (fb), midbrain (mb), hindbrain (hb), and spinal cord (sc). Brain regions are separated by dotted lines. The locus ceruleus (in black) is located just caudal to the midbrain-forebrain boundary and is the major source of spinal NA (González et al., 1994). The mid-hindbrain reticulospinal (Mhr) region (gray) is located dorsally in the caudal hindbrain, with axons that cross the midline (dashed line) and descend to spinal cord, providing a major source of spinal GABAergic inhibition (Roberts et al., 1987). Commissural interneurons (in light gray) cross the midline and provide the major source of spinal glycinergic inhibition (Dale et al., 1986). N0 modulates swimming frequency by indirectly decreasing or increasing endogenous spinal NA release (in black), which in turn modulates spinal glycine release (in light gray) to motor neurons (MN). N0 modulates episode durations by directly modulating GABA release (in gray) to motor neurons. However, the nitrergic modulation of GABA could also affect swimming frequency (see Discussion for details).

prominent role for $\mathrm{NO}$ in this pathway using NO synthase inhibitors, which is consistent with the notion that an endogenous role for NO in mediating GABA release does not appear reliably until larval stages. As Xenopus tadpoles mature, the cement gland stopping pathway degenerates (Boothby and Roberts, 1992a) and becomes incorporated into an endogenous stopping mechanism (Reith and Sillar, 1999). We have suggested previously that NO could be fundamental in the rewiring of this pathway (McLean and Sillar, 2002), given its documented roles in synaptic plasticity (Schuman and Madison, 1994). The findings of Lambert and colleagues (2004) add additional weight to this suggestion, making it likely that inputs other than those deriving from the cement gland must be stimulating NO production. In support, neuronal NO synthase can be coupled postsynaptically to NMDA-type glutamate receptors (Christopherson et al., 1999), suggesting that the generation of $\mathrm{NO}$ could be input specific.

We also suggested that NO may be colocalized with NA in the 
brainstem. In mammals, however, nitrergic neurons in the region of the locus ceruleus are not noradrenergic, but they can regulate the activity of cells in the locus ceruleus that do contain NA (Xu et al., 1998). The same could also be true in Xenopus, in which the nitrergic neurons are simply regulating inputs to the locus ceruleus, thereby controlling spinal glycine release. However, in addition to the effects of NA on swimming frequency, as mentioned previously, NA is also thought to target reticulospinal GABA neurons to shorten episode durations and directly interact with motor neurons to shorten longitudinal delays. If NO is promoting NA release from locus ceruleus projections to the spinal cord, why does SNAP application not also mimic these noradrenergic effects? It is likely that because NO itself has direct effects on GABAergic pathways and motor neuron membrane properties, these effects interfere with the modulatory actions of endogenous NA.

Finally, we cannot rule out the contribution of 5-HT to the effects documented here, given the location of presumed NOgenerating neurons in the raphe region. Although this is a subject for future study, we believe whatever role this interaction may play will be relatively minor, because NO has little effect on motor burst durations, which is the primary effect of bath-applied 5-HT (Sillar et al., 1992). Clearly, more detailed anatomical evidence on the localization of NO synthase with respect to other descending brainstem neurotransmitter systems is now needed.

\section{Nitric oxide and motor control}

In many ways, NO is perfectly suited to metamodulation. First, the generation of NO by neuronal NO synthase (Salerno et al., 1997) and even the transcription of neuronal NO synthase itself (Sasaki et al., 2000) depend on the influx of calcium into the cell. This elegantly ties the generation of NO to cellular activity. Second, given the acknowledged role of $\mathrm{NO}$ as a volume transmitter (Baranano et al., 2001), once generated, NO could diffuse to neighboring neurons and modulate their levels of activity, either by affecting vesicular release of neurotransmitters (Meffert et al., 1996; Micheva et al., 2003), interacting directly with the structure of neurotransmitters (Fossier et al., 1999) or their enzymatic precursors (Kuhn and Arthur, 1997), or activating second messenger pathways via its principle postsynaptic target, guanylate cyclase (Schuman and Madison, 1994). In this way, NO could act as an intrinsic metamodulator (Katz and Frost, 1996) in which the source of NO is a bone fide member of the centers modulating spinal circuitry. Finally, the unique biophysical properties of NO could enable it to synchronize the activity of different networks. For instance, NO has been implicated in the regulation of respiratory activity in amphibians (Hedrick et al., 1998), and NO synthase is located in the medulla where the control circuitry for respiration is located (McLean and Sillar, 2001). Thus, NO could somehow act to link two interrelated functional behaviors, respiration and locomotion (Bramble and Carrier, 1983; Morin and Viala, 2002).

Consistent with the location of NO in brain regions important for motor control (Vincent, 1995), studies impairing the normal production of neuronal NO in mammals have demonstrated dramatic locomotor deficits (Nelson et al., 1995; Dzoljic et al., 1997). However, unlocking the cellular and synaptic mechanisms for such deficits in higher vertebrates is by no means trivial. Thus, one clear advantage of studying animals early in development, such as the Xenopus model, is that their nervous system is functional yet relatively simple. It could be that in mammals, NO is playing a metamodulatory role as described here, regulating fast synaptic inhibition to the spinal cord via aminergic and GABAergic populations.

\section{References}

Baranano DE, Ferris CD, Snyder SH (2001) Atypical neural messengers. Trends Neurosci 24:99-106.

Boothby KM, Roberts A (1992a) The stopping response of Xenopus laevis embryos: behaviour, development and physiology. J Comp Physiol [A] 170:171-180.

Boothby KM, Roberts A (1992b) The stopping response of Xenopus laevis embryos: pharmacology and intracellular physiology of rhythmic spinal neurons and hindbrain neurons. J Exp Biol 169:65-86.

Bramble DM, Carrier DR (1983) Running and breathing in mammals. Science 219:251-256.

Christopherson KS, Hillier BJ, Lim WA, Bredt DS (1999) PSD-95 assembles a ternary complex with the $N$-methyl-D-aspartic acid receptor and a bivalent neuronal NO synthase PDZ domain. J Biol Chem 274:27467-27473.

Dale N (1995) Experimentally derived model for the locomotor pattern generator in the Xenopus embryo. J Physiol (Lond) 489:489-510.

Dale N (2002) Resetting intrinsic purinergic modulation of neural activity: an associative mechanism? J Neurosci 22:10461-10469.

Dale N, Gilday D (1996) Regulation of rhythmic movements by purinergic neurotransmitters in frog embryos. Nature 383:259-263.

Dale N, Roberts A (1985) Dual-component amino-acid-mediated synaptic potentials: excitatory drive for swimming in Xenopus embryos. J Physiol (Lond) 363:35-59.

Dale N, Ottersen OP, Roberts A, Storm-Mathisen J (1986) Inhibitory neurones of a motor pattern generator in Xenopus revealed by antibodies to glycine. Nature 324:255-257.

Dzoljic E, De Vries R, Dzoljic MR (1997) New and potent inhibitors of nitric oxide synthase reduce motor activity in mice. Behav Brain Res 87:209-212.

Fischer H, Merrywest SD, Sillar KT (2001) Adrenoreceptor-mediated modulation of the spinal locomotor pattern during swimming in Xenopus laevis tadpoles. Eur J Neurosci 13:977-986.

Fossier P, Blanchard B, Ducrocq C, LePrince C, Tauc L, Baux G (1999) Nitric oxide transforms serotonin into an inactive form and this affects neuromodulation. Neuroscience 93:597-603.

González A, Marín O, Tuinhof R, Smeets WJ (1994) Ontogeny of catecholamine systems in the central nervous system of anuran amphibians: an immunohistochemical study with antibodies against tyrosine hydroxylase and dopamine. J Comp Neurol 346:63-79.

Harris-Warrick RM, Marder E (1991) Modulation of neural networks for behavior. Annu Rev Neurosci 14:39-57.

Hedrick MS, Morales RD, Parker JM, Pacheco JL (1998) Nitric oxide modulates respiratory-related neural activity in the isolated brainstem of the bullfrog. Neurosci Lett 251:81-84.

Katz PS (1999) Beyond neurotransmission: neuromodulation and its importance for information processing. New York: Oxford UP.

Katz PS, Edwards DH (1999) Metamodulation: the control and modulation of neuromodulation. In: Beyond neurotransmission: neuromodulation and its importance for information processing (Katz PS, ed), pp 349-381. New York: Oxford UP.

Katz PS, Frost WN (1996) Intrinsic neuromodulation: altering neuronal circuits from within. Trends Neurosci 19:54-61.

Kiehn O, Hounsgaard J, Sillar KT (1997) Building blocks in vertebrate central pattern generators. In: Neurons, networks and motor behavior (Stein PSG, Stuart S, Grillner S, Selverston AI, eds), pp 47-59. Boston: MIT.

Kuhn DM, Arthur Jr R (1997) Molecular mechanism of the inactivation of tryptophan hydroxylase by nitric oxide: attack on critical sulfhydryls that spare the enzyme iron center. J Neurosci 17:7245-7251.

Lambert TD, Howard J, Plant A, Soffe S, Roberts A (2004) Mechanisms and significance of reduced activity and responsiveness in resting frog tadpoles. J Exp Biol 207:1113-1125.

Li WC, Perrins R, Walford A, Roberts A (2003) The neuronal targets for GABAergic reticulospinal inhibition that stops swimming in hatchling frog tadpoles. J Comp Physiol [A] 189:29-37.

Lopéz JM, González A (2002) Ontogeny of NADPH diaphorase/nitric oxide synthase reactivity in the brain of Xenopus laevis. J Comp Neurol 445:59-77.

Marder E, Calabrese RL (1996) Principles of rhythmic motor pattern generation. Physiol Rev 76:687-717. 
McDearmid JR (1998) Noradrenergic control of spinal motor circuitry in two related amphibian species: Xenopus laevis and Rana temporaria. $\mathrm{PhD}$ thesis, University of St Andrews.

McDearmid JR, Scrymgeour-Wedderburn JF, Sillar KT (1997) Aminergic modulation of glycine release in a spinal network controlling swimming. J Physiol (Lond) 503:1473-1482.

McLean DL, Sillar KT (2000) The distribution of NADPH-diaphorase labelled interneurons and the role of nitric oxide in the swimming system of Xenopus laevis larvae. J Exp Biol 203:705-713.

McLean DL, Sillar KT (2001) Spatiotemporal pattern of nicotinamide adenine dinucleotide phosphate-diaphorase reactivity in the developing central nervous system of premetamorphic Xenopus laevis tadpoles. J Comp Neurol 437:350-362.

McLean DL, Sillar KT (2002) Nitric oxide selectively tunes inhibitory synapses during vertebrate locomotion. J Neurosci 22:4175-4184.

McLean DL, Sillar KT (2003) Spinal and supraspinal functions of noradrenaline in the frog embryo: consequences for motor behaviour. J Physiol (Lond) 551:575-587.

Meffert MK, Calakos NC, Scheller RH, Schulman H (1996) Nitric oxide modulates synaptic vesicle docking/fusion reactions. Neuron 16:1229-1236.

Merrywest SD, Fischer H, Sillar KT (2002) Alpha-adrenoreceptor activation modulates swimming via glycinergic and GABAergic inhibitory pathways in Xenopus laevis tadpoles. Eur J Neurosci 15:375-383.

Merrywest SD, McDearmid JR, Kjaerulff O, Kiehn O, Sillar KT (2003) Mechanisms underlying the noradrenergic modulation of longitudinal co-ordination during swimming in Xenopus laevis tadpoles. Eur J Neurosci 17:1013-1022.

Mesce KA (2002) Modulation of neuromodulators. Brain Behav Evol 60:337-392

Micheva KD, Buchanan J, Holz RW, Smith SJ (2003) Retrograde regulation of synaptic vesicle endocytosis and recycling. Nat Neurosci 6:925-932.

Morin D, Viala D (2002) Coordinations of locomotor and respiratory rhythms in vitro are critically dependent on hindlimb sensory inputs. J Neurosci 22:4756-4765.

Nelson RJ, Demas GE, Huang PL, Fishman MC, Dawson VL, Dawson TM, Snyder SH (1995) Behavioural abnormalities in male mice lacking neuronal nitric oxide synthase. Nature 378:383-386.

Nieuwkoop PD, Faber J (1956) Normal tables for Xenopus laevis. Amsterdam: Daubin.

Nusbaum MP, Blitz DM, Swensen AM, Wood D, Marder E (2001) The roles of co-transmission in neural network modulation. Trends Neurosci 24:146-154.

Perrins R, Roberts A (1995) Cholinergic and electrical motoneuron-tomotoneurons synapses contribute to on-cycle excitation during swimming in Xenopus embryos. J Neurophysiol 73:1005-1112.

Perrins R, Walford A, Roberts A (2002) Sensory activation and role of inhibitory reticulospinal neurones that stop swimming in hatchling frog tadpoles. J Neurosci 22:4229-4240.

Reith CA, Sillar KT (1997) Pre- and postsynaptic modulation of spinal GABAergic neurotransmission by the neurosteroid, $5 \beta$-pregnan- $3 \alpha$-ol20-one. Brain Res 770:202-212.

Reith CA, Sillar KT (1999) Development and role of $\mathrm{GABA}_{\mathrm{A}}$ receptormediated synaptic potentials during swimming in postembryonic Xenopus laevis tadpoles. J Neurophysiol 82:3175-3187.
Roberts A (1990) How does a nervous system produce behaviour? A case study in neurobiology. Sci Prog 74:31-51.

Roberts A, Alford ST (1986) Descending projections and excitation during fictive swimming in Xenopus embryos: neuroanatomy and lesion experiments. J Comp Neurol 250:253-261.

Roberts A, Clarke JDW (1982) The neuroanatomy of an amphibian embryo spinal cord. Philos Trans R Soc Lond B Biol Sci 296:195-212.

Roberts A, Dale N, Ottersen OP, Storm-Mathisen J (1987) The early development of neurons with GABA immunoreactivity in the CNS of Xenopus laevis embryos. J Comp Neurol 261:435-449.

Salerno JD, Harris DE, Irizarry K, Patel B, Morales AJ, Smith SM, Martaseki P, Roman LJ, Masters BS, Jones CL, Weissman BA, Lane P, Liu Q, Gross SS (1997) An autoinhibitory control element defines calcium regulated isoforms of nitric oxide synthase. J Biol Chem 272:29769-29777.

Sasaki M, Gonzalez-Zulueta M, Huang H, Herring WJ, Ahn S, Ginty DD, Dawson VL, Dawson TM (2000) Dynamic regulation of neuronal NO synthase transcription by calcium influx through a CREB family transcription factor-dependent mechanism. Proc Natl Acad Sci USA 97:8617-8622.

Schuman EM, Madison DV (1994) Nitric oxide and synaptic function. Annu Rev Neurosci 17:153-183.

Sillar KT, Roberts A (1993) Control of frequency during swimming in Xenopus embryos: a study on interneuronal recruitment in a spinal rhythm generator. J Physiol (Lond) 472:557-572.

Sillar KT, Wedderburn JF, Simmers AJ (1992) Modulation of swimming rhythmicity by 5-hydroxytryptamine during post-embryonic development in Xenopus laevis. Proc R Soc Lond B Biol Sci 250:107-114.

Sillar KT, Kiehn O, Kudo N (1997) Chemical modulation of vertebrate motor circuits. In: Neurons, networks and motor behavior (Stein PSG, Stuart S, Grillner S, Selverston AI, eds), pp 183-193. Boston: MIT.

Sillar KT, McLean DL, Merrywest SD, Fischer H (2002) Fast inhibitory synapses: targets for neuromodulation and development of vertebrate motor behaviour. Brain Res Brain Res Rev 40:130-140.

Simmers J, Meyrand P, Moulins M (1995) Modulation and dynamic specification of motor rhythm-generating circuits in crustacea. J Physiol (Paris) 89:195-208.

Soffe SR (1987) Ionic and pharmacological properties of reciprocal inhibition in Xenopus embryo motoneurones. J Physiol (Lond) 382:463-473.

Svensson E, Grillner S, Parker D (2001) Gating and braking of short- and long-term modulatory effects by interactions between colocalized neuromodulators. J Neurosci 21:5984-5992.

Thoby-Brisson M, Simmers J (1998) Neuromodulatory inputs maintain expression of a lobster motor pattern-generating network in a modulationdependent state: evidence from long-term decentralization in vitro. J Neurosci 18:2212-2225.

van Mier P, Joosten HW, van Reden R, ten Donkelaar HJ (1986) The development of serotonergic raphespinal projections in Xenopus laevis. Int J Dev Neurosci 4:465-476.

Vincent SR (1995) Localization of nitric oxide neurons in the central nervous system. In: Nitric oxide in the nervous system (Jenner P, ed), pp 83-102. New York: Academic.

Xu ZQ, de Vente J, Steinbusch H, Grillner S, Hokfelt T (1998) The NOcGMP pathway in the rat locus coeruleus: electrophysiological, immunohistochemical and in situ hybridization studies. Eur J Neurosci 10:35083516. 